\title{
Urinary tract stone surgery in patients with urinary diversion and vesicostomy: a single center experience
}

\author{
๑1 Kubilay Sarıkaya, @Çağrı Şenocak, ๑Fahri Erkan Sadioğlu, ®Mehmet Çiftçi,®Ömer Faruk Bozkurt \\ University of Health Sciences, Keçiören Training and Research Hospital, Department of Urology, Ankara, Turkey
}

Cite this article as: Sarıkaya K, Şenocak Ç, Sadioğlu FE, Çiftçi M, Bozkurt ÖF. Urinary tract stone surgery in patients with urinary diversion and vesicostomy: a single center experience. J Health Sci Med 2021; 4(1): 78-83.

\begin{abstract}
Objective: To report our experience in percutaneous nephrolithotomy and endoscopic urinary tract stone surgery in patients with urinary diversion or vesicostomy.

Material and Method: Data of 21 patients with urinary diversion or vesicostomy who underwent surgery for urinary tract stones in our clinic between January 2008 and January 2020 were retrospectively analyzed. Eight patients (38\%) underwent percutaneous nephrolithotomy, 2 patients (9.5\%) underwent antegrade flexible ureteroscopy, 4 patients (19.0\%) underwent retrograde semi-rigid or flexible ureteroscopy, 5 patients (23.8\%) underwent retrograde pouch lithotripsy and 2 patients (9.5\%) underwent percutaneous cystolithotripsy with vesicostomy tract entrance. Preoperative and postoperative data of the patients were evaluated.

Results: The male to female ratio was $16 / 5$.The mean age of the patients was $54.6 \pm 10.1$ years and mean preoperative stone diameter was $2.8 \pm 4.5 \mathrm{~cm}$. It was determined that 14 patients $(66.6 \%)$ had ileal conduit (Bricker anastomosis), 5 patients (23.8\%) had ureterocutaneostomy, and 2 patients (9.5\%) had vesicostomy. Stone-free rate was $85.7 \%$ after single session of treatment. In the postoperative period, febrile urinary tract infection was observed in $4(19.0 \%)$ patients, urinary system obstruction secondary to stone in $3(14.2 \%)$ patients and anastomotic leakage in 1 (4.7\%) patient.

Conclusion: Percutaneous nephrolithotomy, antegrade ureterorenoscopy, retrograde ureterorenoscopy and vesicostomy entry cystolithotripsy are highly effective and safe methods in patients with urinary diversion and vesicostomy. The most important factors affecting the success are the experience of surgical team that can apply procedural options together with careful preoperative preparation and correct instrumentation.
\end{abstract}

Keywords: Urinary diversion, stone formation, stone surgery

\section{INTRODUCTION}

Ureterosigmoidostomy procedure was first applied after radical cystectomy by Simon et al. (1) and following this, different types of urinary diversion such as cutaneous conduit, orthotopic neobladder and continent urinary diversion were developed. Stone formation in the upper urinary tract as well as in the reservoir or conduit is one of the most common complications in patients with urinary diversion (2). The incidence of stone formation in patients with ileal conduit was reported to be from $9 \%$ to $11 \%$, while it was reported that $17 \%$ patients with Koch pouch and from $11 \%$ to $12.9 \%$ of patients with Indiana pouch developed urinary tract stones in the long term follow-up period (3-5). Similar to patients with urinary diversion, it is known that both bladder stones and upper urinary tract stones are frequently develop in patients with neurogenic bladder developing secondary to spinal cord injury (6).

Stone surgery in patients with urinary diversion presents various difficulties for urologists. Difficulties in visualizing the ureter orifices through the pouch and entering the ureter during both imaging and retrograde approach due to the impaired anatomical structure constitute the main problem in this area (7-9). On the other hand, stone recurrence reported in $33 \%$ to $63 \%$ of patients with urinary diversion within 3-5 years after the first surgical intervention significantly limits the option of the open stone surgery (10). Therefore, in patients with urinary diversion, percutaneous nephrolithotomy (PNL), semi-rigid or flexible antegrade ureteroscopy (URS) performed through percutaneous tract, or 
combined antegrade and retrograde approaches constitute the preferred surgical options (11). Although successful results of extracorporeal shoch wave lithotripsy (ESWL) have been reported in stone patients with urinary diversion in stones smaller than $2 \mathrm{~cm}$, it is known that the success rate of ESWL decreases in larger stones (10).

As the literature data and our clinical experience indicate, urinary system stone stone surgery in patients with urinary diversion and vesicostomy requires preoperative instrumentation preparation and surgical team experience, which allows all options to be applied during the operation.

In this study, we aimed to share our experiences in urinary system stone surgeries performed in patients with urinary diversion and vesicostomy in our clinic, which is one of the centers working intensively on urinary system stone surgery.

\section{MATERIAL AND METHOD}

The study was carried out with the permission of Keçiören Training and Research Hospital Health Application Research Center Medical Specialty Education Board (Date: 08.12.2020 IRB: 2012-KAEK-15/2202) of our institution, the data of 21 patients with urinary diversion or vesicostomy who underwent surgical intervention for urinary tract stones between January 2008 and January 2020 were retrospectively analyzed. All procedures were performed adhered to the ethical rules and the Helsinki Declaration of Principles.

During the preoperative preparation period, all patients were evaluated with routine preoperative blood tests, urine analysis, urine culture, and non-contrast abdominal computed tomography (CT). Intravenous urography (IVU) was performed to evaluate the anatomical structure of kidney. Possible anatomical variations were evaluated according to the type of operation and diversion the patients had, and the possible difficulties to be encountered during the operation were discussed and necessary and sufficient instrumentation was provided according to these possibilities. In the preoperative evaluation, it was determined that $14(66.6 \%)$ patients had Bricker-type ileal conduit diversion, 5 (23.5\%) had ureterocutaneostomy performed during radical cystectomy, and 2 (9.5\%) patients had vesicostomy due to neurogenic bladder developing secondary to spina bifida. Appropriate prophlylactic and therapeutic antibiotic was administered to patients according to preoperative urine culture results. Intravenous $1 \mathrm{~g} 3^{\text {rd }}$ generation cephalosporin was administered to all patients for preoperative prophylaxis. The operations included in the study were performed by a total of six surgeons working in the same center.

\section{Surgical Procedures}

Percutaneus nephrolithotomy (PNL): All patients with renal stones were evaluated primarily in lithotomy position in terms of performing retrograde ureteral catheterization and, if possible, retrograde intrarenal surgery (RIRC). Patients whose ureter orifices cannot be seen and retrograde access was not possible were prepared for percutaneous intervention, a 16-18 fr foley catheter was inserted into the pouch from the stoma, the balloon was inflated with 5-6 cc saline, and the patient was turned to the prone position. After the appropriate position was given, radiopaque liquid diluted with $50 \%$ saline was given into the pouch through the foley catheter, and it was tried to pass from the ureter to the kidney to visualize the renal collecting system. In cases which sufficient radiopaque material could not reached into the kidney and the renal collecting system, the ultrasound-guided entry technique was used. An 18-gauge percutaneous needle was used to enter the renal collecting system, and the needle was removed so that the outer sheath remained in the renal collecting system by providing the targeted renal calix entry. Afterwards, radiopaque fluid was injected into the renal collecting system through the outer sheath of the percutaneous needle, and the collecting system was visualized, and the entrance location and anatomical structure were evaluated. In cases where it was seen that proper calix entry could not be achieved, re-entry was made with a second needle and the outer sheath of the first entry needle was not removed and used for radiopaque fluid infusion into the renal collecting system in order to ensure adequate visualization. After proper renal calix insertion was provided, a 0.035 -inch hydrophilic guide wire was advanced into the renal collecting system through the outer sheath of the needle, followed by percutaneous dilatation with percutaneous dilatators ranging from $24 \mathrm{fr}-30 \mathrm{fr}$, allowing percutaneous access to the renal collecting system with rigid nephroscope (Karlstorz ${ }^{\oplus}-22 \mathrm{fr}$ ). Following the percutaneous entry, the stone was fragmented and removed with use of an ultrasonic lithotripter $\left(\mathrm{EMS}^{\circledR}\right)$, or pneumatic lithotripter $\left(\mathrm{EMS}^{\circledR}\right)$. A flexible cystoscope (Olympus-21 fr) was used in some calix stones that cannot be reached with rigid nephroscope. Following the procedure, a 16-fr catheter was placed in all patients as a nephrostomy, and the catheter had placed in the pouch was removed.

Antegrade URS: Antegrade URS procedure was used in upper ureteral stones where retrograde intervention was not possible and for stones that could not be reached with a rigid or flexible nephroscope. At the entrance, the same procedure was applied as the PNL entry and percutaneous entry to the kidney through the appropriate calix was provided. Then, a 0.035 -inch hydrophilic guide wire was advanced antegradely into the ureter and then the stone was reached by entering the ureter with 
a flexible ureteroscope (Olympus $-9.5 \mathrm{fr}$ ) through the nephrostomy tract. The stone was fragmented with using a $200 \mu \mathrm{m}$ or $500 \mu \mathrm{m}$ Ho:YAG laser energy (StoneLight ${ }^{\circledR}$ ) and removed with a $2.2 \mathrm{fr}$ nitinol stone basket. After the stone was removed, imaging was performed by antegrade ureterography in terms of possible obstruction and anostomosis integrity, and a $5 \mathrm{fr}$ doule-j stent was placed through the nephrostomy tube up to the reservoir pouch. Subsequently, a 16 fr nephrostomy catheter was inserted and the procedure was terminated. The nephrostomy tube was removed on the postoperative day 3 , and then on the $7^{\text {th }}$ day, the duoble-j stend was removed through the pouch using a rigit (Karl-Storz ${ }^{\circledR}-17$ fr) or flexible cystoscope.

Retrograde URS: Except for patients with vesicostomy, ureteral catheterization for PNL or URS was tried in all 19 patients with urinary diversion, but only in $4(21.0 \%)$ of the patients procedure wassuccessful. Retrograde URS was performed in cases that the ureter orifice can be seen through the diversion pouch. Using a $17 \mathrm{fr}$ rigit cystoscope or 9.5-fr semi-rigid ureterorenoscope (Olymus ${ }^{\otimes} 9.5 \mathrm{fr}$ ), a 0.035 -inch hydrophilic guidewire was advanced to the ureter under direct visualition of the ureter orifice of the stone side. Subsequently, the ureter was entered by using a semi-rigid or flexible ureterorenoscope under the guidance of a hydrophilic guidewire. The stone was fragmented with a $200 \mu \mathrm{m}$ or $500 \mu \mathrm{m}$ Ho: YAG laser energy and removed using a $2.2 \mathrm{fr}$ nitinol stone basket. At the end of the procedure, a 5 fr double-j stent was placed over the hydrophilic guidewire into the ureter and the procedure was terminated.The double-j stent was removed on the postoperative day 7 by retrograde route using a rigit or flexible cystoscope.

Retrograde pouch lithotripsy with stomal entry: Retrograde pouch lithotripsy with stomal entry was applied in patients with reservoir stones. For this purpose, the pouch was inserted retrograde from the stoma with using a $22 \mathrm{fr}$ nephroscope and the stone was fragmented with a pneumatic or ultrasonic lithotriptor and removed with the aid of a stone basket. During the procedure, the presence of stone fragments between the mucosal folds was checked by fluroscopy, and possible fragmented stones were removed. At the end of the procedure, radiopaque fluid was injected into the pouch and the presence of possible anastomotic leakage was checked, and at the end of the procedure, a 16-18 fr foley catheter was placed in the pouch and the procedure was terminated. The foley catheter in the pouch was removed on the second postoperative day.

Percutaneous cystolithotripsy with vesicostomy entry: In patients with vesicostomy, which was performed due to neurogenic bladder developing secondary to spina bifida, percutaneous cystolithotripsy with vesicostomy entry was applied for bladder stone. In this procedure, the bladder was entered through the vesicostomy tract with a $22-\mathrm{fr}$ nephroscope, and then the stone was fragmented using an ultrasonic or pneumatic lithotripter and removed using a stone basket. When necessary, $200 \mu \mathrm{m}$ or $500 \mu \mathrm{m}$ Ho: YAG laser energy was also used for stone fragmentation. At the end of the procedure, a 16-18 fr foley catheter was inserted through the vesicostomy tract into the bladder and removed on the second postoperative day.

\section{Statistical Analysis}

All statistical analyses were performed with SPSS 24.0 (IBM Corp., Chicago) for Windows. The mean \pm standard deviation was used for parametric data and the median and minimum-maximum values were used for nonparametric data.

\section{RESULTS}

The male to female ratio was $16 / 5$. The mean age of the patients was $54.6 \pm 10.1(16-71)$ years. The most common comorbidity factor detected in the preoperative period was hypertension which was seen in 14 (66.6\%) patients. The mean preoperative creatinine level was $1.1 \pm 0.3$ (80.72.2) $\mathrm{mg} / \mathrm{dL}$. The most common stone localization was kidney in 11 (52.3\%) patients, while it was found in the reservoir pouch in $5(23.8 \%)$ patients and in the ureter in $3(14.2 \%)$ patients. Bladder stones were detected in the other $2(9.5 \%)$ patients with vesicostomy included in the study. Preoperative patient characteristics are shown in Table 1.

\begin{tabular}{|lc|}
\hline Table 1. Preoperative patient's characteristics & \\
\hline Age, mean \pm SD (minimum-maximum),years & $54.6 \pm 10.1(16-71)$ \\
Gender (n,\%) & - \\
Male & $16(76.1 \%)$ \\
Female & $5(23.8 \%)$ \\
Comorbidities (n,\%) & - \\
Hypertension & $14(66.6 \%)$ \\
Diabetes mellitus & $4(19.0 \%)$ \\
Dyslipidemia & $5(23.8 \%)$ \\
Chronic obstructive pulmonary disease & $2(9.5 \%)$ \\
Rheumatoid arthritis & $1(4.7)$ \\
Preoperative serum creatinine level (mg/dL) & $1.1 \pm 0.3(0.7-2.2)$ \\
Stone localization (n,\%) & - \\
Kidney & $11(52.3 \%)$ \\
Ureter & $3(14.2 \%)$ \\
Reservoirpouch & $5(23.8 \%)$ \\
Bladder & $2(9.5 \%)$ \\
Stone size (cm) & $2.8 \pm 4.5(1.4-8.5)$ \\
Diversion type (n,\%) & - \\
İleal conduit (Bricker type) & $14(66.6 \%)$ \\
Ureterocutaneostomy & $5(23.8 \%)$ \\
Vesicostomy & $2(9.5 \%)$ \\
\hline
\end{tabular}


It was observed that the mean operation time was $74.5 \pm 14.6$ (40-130) minutes and the most common operationtype was PNLthat was performed in 8 (38.0\%) patients. Other procedures performed were antegrade URS in $2(9.5 \%)$ patients, retrograde URS in 4 patients (19.0\%), retrograde pouch lithotripsy with stomal entry in 5 patients $(23.8 \%)$ and percutaneous cystolithotripsy with vesicostomy entry in 2 patients (9.5\%). In the early postoperative period, febrile urinary tract infection was developed in $4(19.0 \%)$ patients. In total of $3(14.2 \%)$ patients, 2 (9.5\%) who underwent PNL and 1 (4.7\%) who underwent antegrade URS, urinary obstruction was developed due to residual stones in the postoperative period and secondary surgical intervention was required. Minimal anastomotic leakage was found at the end of the procedure in $1(4.7 \%)$ of the patients who underwent retrograde URS, and it was observed that the urinary leakage spontaneously recovered by a double-j stent without additional surgical intervention. Postoperative findings of the patients are shown in Table 2.

\section{Table 2. Surgical procedures and postoperative results}

Surgical procedure $(n, \%)$

Percutaneus nephrolithotomy

$8(38.0 \%)$

Antegradeureteroscopy (flexible)

$2(9.5 \%)$

Retrogradeureteroscopy (semi-rigid or flexible) $\quad 4(19.0 \%)$

Retrograde pouch lithotripsy (transstomal) $5(23.8 \%)$

Percutaneus cystolithotripsy (vesicostomy-entry) $2(9.5 \%)$

Operation time (minutes)

$74.5 \pm 14.6(40-130)$

Postoperative serum creatinine level (mg/dL)

$0.8 \pm 0.4(0.7-1.9)$

Complication ( $\mathrm{n}, \%)$

Febrile urinary tract infection

$4(19.0 \%)$

Urinary obstruction and secondary surgery

$3(14.2 \%)$

Anastomotic urine leakage

$1(4.7 \%)$

Stone composition $(\mathrm{n}, \%)$

Calcium-oxalate

$9(42.8 \%)$

Struvite

$10(47.6 \%)$

Calcium-phosphate

$1(4.7 \%)$

Urine-acite

$1(4.7 \%)$

\section{DISCUSSION}

The most common postoperative complication seen in patients with urinary diversion is urinary stone formation with a prevalence of $2.6 \%$ to $15.3 \%(12,13)$. The main cause of urinary system stone disease in these patients is the presence of increased chronic infection and metabolic changes, as well as the structural and mechanical differentiation in the urinary system (14). Stasis in the upper urinary system and the presence of hydronephrosis developing secondary to this, together with the change of the anatomical structure as a result of urinary diversion, are seen with a frequency of up to $80 \%$ in these patients (15). Mucus production originating from the intestinal mucosa and the foreign body reaction caused by it, stomal stenosis and urinary retention are also important factors that cause stone formation in the pouch (16). Metabolic changes are characterized by systemic acidosis, hypercalciuria, hyperoxaluria, and hypocitraturia (17). These factors increase the risk of calcium-containing stones (17). However, the most common factor causing stone formation in patients with urinary diversion is the presence of increased chronic infection (18). Therefore, struvite stones are the most common stone formation in patients with urinary diversion. In our study, similar to the literature, struvite stones were found to be the most common stone formation.

In patients with urinary diversion with asymptomatic small size urinary stone, ESWL is recommended as a non-invasive initial treatment option in order to reduce potential surgical difficulties and complication (19). Ahmed et al. (20) reported that they were performed ESWLin 27 patients with urinary diversion with stones smaller than $1 \mathrm{~cm}$. According to this study, the success rate of upper urinary tract stones in urinary diversion patients with single session ESWL treatment was reported as $81.5 \%(22 / 27)$. However, in this study, it was also reported that the stone-free status could not completely reached in $2(7.4 \%)$ patientsand $1(3.7 \%)$ had a significant residual stone, $2(7.4 \%)$ patients developed renal obstruction after ESWL and 5 (18.5\%) patients had PNL, antegrade URS or a secondary procedure such as open operation is required. The fact that the stone fragments after ESWL enters into the intestinal folds in the pouch and do not drain spontaneously and cause the formation of large stones again by undergoing reformation is considered as an important factor limiting the effectiveness of this procedure, especially in stones larger than $1 \mathrm{~cm}$ (18-20).

Percutaneous nephrolithotomy is one of the most important options in the surgical treatment of large stones in the upper urinary system in patients with urinary diversion, and its success rate has been reported to be from $60 \%$ to $80 \%$ (21). In a study conducted by Lindsay et al. (22) involving 77 patients, the effectiveness of PNL, retrograde URS and ESWL in the treatment of upper urinary tract stones in patients with urinary diversion was compared. In this study, it was stated that the average stone size in the PNL group was significantly higher than the URS and ESWL groups $(2.1$ vs 0.9 and $1.0 \mathrm{~cm}$,respectively, $\mathrm{p}<0.0001)$.In this study, although the mean stone size was significantly larger, the total stone-free rate was significantly better in PNL than the URS and ESWL groups ( $83.3 \%$ vs $33.3 \%$ and $30 \%$, respectively, $\mathrm{p}<0.0001)$. Total complication rates were reported to be similar between the groups $(p=0.900)$. In another similar study by Zhong et al. (23) including 20 patients with urinary diversion, 8 patients underwent PNL, 3 patients had antegrade URS, 6 patients had percutaneous pouch lithotripsy, 2 patients 
had transurethral neo-bladder lithotripsy, 1 patient had open stone surgery. It was reported that 18 (90\%) patients werestone-free at the end of the procedures. According to this study, an insignificant residual stone remained in 1 (5\%) patient who underwent PNL and 1 (5\%) patient who underwent percutaneous pouch surgery, and only 2 (15\%) patients had postoperative fever and 1 (5\%) patient had postoperative urinary extravasation. In our study, it was seen that the most common surgical procedure used in patients with urinary diversion was PNL, and similar to the literature, a high rate of stone-free was achieved. In our study, secondary intervention was required due to urinary obstruction in only 1 of the patients who underwent PNL. This result supports the idea that PNL should be the preferred surgical method for the surgical treatment of upper urinary tract stones in patients with urinary diversion due to its high success rate. The most common problem encountered when performing PNL in patients with urinary diversion is that the retrograde catheterization cannot be performed most of the time due to the difficulty in visualizing the ureter orifices through the pouch (17). Ultrasonographic approach can be used in these patients for achieving proper access to the collecting system (24). At this stage, proper preoperative preparation and equipment competence, as well as surgical team experience come to the fore. In our study, there was no access problem in any urinary diversion patient who underwent PNL, and therefore, there was no need to switch to another surgical method.

The most important problem with retrograde URS in patients with urinary diversion is the difficulty in identifying the ureteral orifices within the reservoir $(3,4,19,20,23)$. Singla N et al. (25) reported the results of retrograde URS intervention performed in 45 neobladder patients due to several upper urinary tract anomalies. According to this study, it was reported that retrograde URS intervention was successful in 2 of 4 urinary system stone patients. In the study of Delveccio et al. (11) it was reported that retrograde URS can be performed more easily under the guidance of the guidewire advanced into the neo-bladder from antegrade route, but the procedure is very time consuming. Therefore, antegrade URS is recommended for upper ureteral stones or renal calix stones that cannot be reached with PNL in urinary diversion patients, and the procedure can be successfully performed with a semi-rigid or flexible ureterorenoscope (26). In our study, retrograde URS intervention was largely unsuccessful because the ureteral orifices could not be clearly visualized so the procedure changed to antegrade URS or PNL in these patients. This result indicates that the antegrade URS or PNL should be the more preferred choice rather than retrograde URS in patients with urinary diversion with upper urinary tract stones due to the loss of time and effort.
In several studies, it has been reported that stones in the diversion pouch can be successfully treated with transurethral lithotripsy, percutaneous pouch lithotripsy or stomal entry pouch lithotripsy in patients with both orthotopic urinary diversion and cutaneous urinary diversion $(7,11,15)$. The surgical method to be chosen depends on the type of diversion and the size of the stone. In our study, stomal entry pouch lithotripsy was applied to all patients with stones in the reservoir pouch and complete stone-free was achieved in all patients without complications, similar to the literature.

Various studies have reported that the risk of bladder stone formation is increased in neurogenic bladder disorder that develops in patients with spinal cord injury (27). Ordet al. (28) reported that the risk of bladder stone formation was significantly increased in patients with spinal cord injury, both in patients who underwent intermittent urethral catheterization, and in patients with vesicostomy and cystostomy. In our study, bladder stones were detected in 2 patients who had vesicostomy due to neurogenic bladder secondary to spina bifida, and complete stone-free was achieved with vesicostomy-entry cystolithotripsy.

\section{Limitations}

The most important limitation of our study is its retrospective nature. In addition, the fact that urinary system stone surgery is one of the rare cases in patients with urinary diversion and therefore the low number of patients in the study can be considered as another limitation. The fact that no comparison was made due to the absence of a control group in our study can be considered as another limitation.

\section{CONCLUSION}

Surgical treatment of urinary tract stones in patients with urinary diversion and vesicostomy varies according to the type of diversion, stone location and stone size. In upper urinary tract stones, antegrade URS and PNL should be the primary choice due to their short operation time and effort advantage. Retrograde URS should be preferred in patients where ureter orifices can be clearly visualized through the diversion pouch. Stones in diversion pouch can be easily treated with both stomal entry and percutaneous stomal entry, and high stone-free rate can be achieved. Surgical treatment of bladder stones in patients with vesicostomy can be successfully performed with vesicostomy entry. The most important factors in the success of urinary tract stone surgery in patients with urinary diversion and vesicostomy are the correct management of the preoperative surgical preparation process and instrumentation preparation in which all interventions can be performed, as well as surgical team experience. 


\section{ETHICAL DECLARATIONS}

Ethics Committee Approval: The study was carried out with the permission of Keçiören Training and Research Hospital Health Application Research Center Medical Specialty Education Board (Date: 08.12.2020 IRB: 2012KAEK-15/2202).

Informed Consent: Because the study was designed retrospectively, no written informed consent form was obtained from patients.

Referee Evaluation Process: Externally peer-reviewed.

Conflict of Interest Statement: The authors have no conflicts of interest to declare.

Financial Disclosure: The authors declared that this study has received no financial support.

Author Contributions: All of the authors declare that they have all participated in the design, execution, and analysis of the paper, and that they have approved the final version.

\section{REFERENCES}

1. Simon J. Extrophia vesicae (absence of the anterior walls of the bladder and pubic abdominal parietes); operation for directing the orifices of the ureters into the rectum; temporary success; subsequent death; autopsy. Lancet 1852; 2: 568.

2. Urh A, Soliman PT, Schmeler KM, et al. Postoperative outcomes after continent versus incontinent urinary diversion at the time of pelvic exenteration for gynecologic malignancies. Gynecol Oncol 2013; 129: 580-5.

3. Turk TM, Koleski FC, Albala DM. Incidence of urolithiasis in cystectomy patients after intestinal conduit or continent urinary diversion. World J Urol 1999; 17: 305-7.

4. Ginsberg D, Huffman JL, Lieskovsky G, Boyd S, Skinner DG. Urinary tract stones: a complication of the Kock pouch continent urinary diversion. J Urol 1991; 145: 956-9.

5. Arai Y, Kawakita M, Terachi T, et al. Long-term follow up of the Kock and Indiana pouch procedures. J Urol 1993; 150: 51-5.

6. Ku JH, Jung TY, Lee JK, Park WH, Shim HB. Risk factors for urinary stone formation in men with spinal cord injury: a 17-year follow-up study. BJU Int 2006; 97: 790-3.

7. Okhunov Z, Duty B, Smith AD, Okeke Z. Management of urolithiasis in patients after urinary diversions. BJU Int 2011; 108: 330-6.

8. Fernandez A, Foell K, Nott L, Fernandez A. Percutaneous nephrolithotripsy in patients with urinary diversions: A casecontrol comparison of perioperative outcomes. J Endourol 2011; 25: $1615-8$.

9. Franzoni DF, Decter RM. Percutaneous vesicolithotomy: an alternative to open bladder surgery in patients with an impassable or surgically ablated urethra. J Urol 1999; 162: 777-8.

10. Cohen TD, Streem SB, Lammert G. Long-term incidence and risks for recurrent stones following contemporary management of upper tract calculi in patients with a urinary diversion. J Urol1996; 155: 62-5.

11.Delvecchio FC, Kou RL, Iselin CE, et al: Combined antegrade and retrograde endoscopic approach for management of urinary diversion-associated pathology. J Endourol 2000; 14: 251-6.
12. Terai A, Arai Y, Kawakita M et al. Effect of urinary intestinal diversion on urinary risk factors for urolithiasis. J Urol 1995; 153: 37.

13. Shimko MS, Tollefson MK, Umbreit EC, et al. Long-term complications of conduit urinary diversion. J Urol 2011; 185: 562.

14. Beiko DT and Razvi H: Stones in urinary diversions: update on medical and surgical issues. Curr Opin Urol 2002; 12: 297.

15.el-Nahas AR, Eraky I, el-Assmy AM, et al. Percutaneous treatment of large upper tract stones after urinary diversion. Urology 2006; 68: 500-4.

16. Haselhuhn GD, Kropp KA, Keck RW, Selman SH. Photochemical ablation of intestinal mucosa for bladder augmentation. J Urol. 1994; 152: 2267-71.

17. Cohen TD, Streem SB, and Lammert GK: Selective minimally invasive management of calculi in patients with urinary diversions. J Urol 1994; 152: 1091-4.

18. Razvi HA, Martin TV, Sosa ER, et al. Endourologic management of complications of urinary intestinal diversion. AUA Update Series 11(lesson 22): 174 -9, 1996.

19. Cass AS, Lee JY, Aliabadi.Extracorporeal shock wave lithotripsy and endoscopic management of renal calculi with urinary diversions. J Urol 1992; 148: 1123-5.

20.El-Assmy A, El-Nahas AR, Mohsen T, et al. Extracorporeal shock wave lithotripsy of upper urinary tract calculi in patients with cystectomy and urinary diversion. Urology 2005; 66: 510-3.

21. Madbouly K. Large orthotopic reservoir stone burden: Role of open surgery. Urol Ann 2010; 2: 96-9.

22.Hertzig LL, Iwaszko MR, Rangel LJ, Patterson DE, Gettman MT, Krambeck AE. Urolithiasis after ileal conduit urinary diversion: a comparison of minimally invasive therapies. J Urol 2013; 189: 2152-7.

23.Zhong W, Yang B, He F, Wang L, Swami S, Zeng G. Surgical management of urolithiasis in patients after urinary diversion. PLoS One 2014; 31: 9.

24.Desai M. Ultrasonography-guided punctures-with and without puncture guide. J Endourol 2009; 23: 1641-3.

25. Singla N, Montie JE, Lee CT, Wolf JS Jr, Faerber GJ. Experience with 45 Consecutive Patients with Neobladders Undergoing Retrograde Ureteroscopy for Upper Tract Abnormalities. Urol Pract 2015; 2: 244-9.

26.Li X, He Z, Wu K, Li SK, Zeng G. Chinese minimally invasive percutaneous nephrolithotomy: the Guangzhou experience. J Endourol 2009; 23: 1693-7.

27. Yamamoto M, Kashiwai H, Hirayama A, et al. [Long-term followup of female tetraplegic patients with cutaneous vesicostomy]. Hinyokika Kiyo1997; 43: 263-6.

28. Ord J, Lunn D, Reynard J. Bladder management and risk of bladder stone formation in spinal cord injured patients. J Urol 2003; 170: 1734-7. 